\title{
Autophagy protects nucleus pulposus cells from cyclic mechanical tension-induced apoptosis
}

\author{
MINGHUI YANG ${ }^{1}$, CHENCHENG FENG $^{1}$, YANG ZHANG ${ }^{1}$, CHANG LIU $^{1}$,

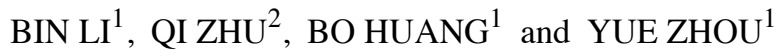 \\ ${ }^{1}$ Department of Orthopedics, Xinqiao Hospital, Third Military Medical University; ${ }^{2}$ Medical Research Center, \\ Southwestern Hospital, Third Military Medical University, Chongqing 400037, P.R. China
}

Received February 11, 2019; Accepted May 24, 2019

DOI: $10.3892 /$ ijmm.2019.4212

\begin{abstract}
Intervertebral disc (IVD) degeneration (IDD) is considered to be a primary cause of lower back pain. Mechanical stress is one of the most important factors affecting IDD. It has been demonstrated that apoptosis is important in the decrease of functional IVD cells, and that mechanical stress influences disc cell apoptosis. Autophagy, an adaptive response of the cells to survival when faced with different conditions of stress, has been documented in IDD. Apoptosis and autophagy share the same stimuli and regulatory proteins, but have different threshold responses. Recently, cyclic mechanical tension (CMT) has been shown to influence IVD cell apoptosis and autophagy. However, the conversion and coordination between apoptosis and autophagy induced by CMT remains to be fully elucidated. In the present study, it was found that CMT with $20 \%$ elongation generated by the Flexercell Tension system induced the apoptosis of nucleus pulposus (NP) cells in a time-dependent manner. When the cells were stretched for $>6 \mathrm{~h}$, autophagy was increased, and showed a tendency to decrease with the duration of CMT. The autophagic activity of NP cells was partially decreased by 3-MA and was not significantly regulated by rapamycin. CMT-induced apoptosis was partially enhanced by the decreased autophagic activity induced by 3-MA. In addition,
\end{abstract}

Correspondence to: Dr Bo Huang or Dr Yue Zhou, Department of Orthopedics, Xinqiao Hospital, Third Military Medical University, 183 Xinqiao Main Street, Chongqing 400037, P.R. China

E-mail: fmmuhb@126.com

E-mail: happyzhou@vip.163.com

Abbreviations: IVD, intervertebral disc; IDD, intervertebral disc degeneration; CMT, cyclic mechanical tension; NP, nucleus pulposus; ROS, reactive oxygen species; LBP, lower back pain; AF, annulus fibrosus; PCD, programmed cell death; RT-qPCR, reverse transcription-quantitative polymerase chain reaction; MFI, mean fluorescence intensity; MDC, monodansylcadaverine; 7-AAD, 7-amino-actinomycin D

Key words: nucleus pulposus cell, cyclic mechanical tension, apoptosis, autophagy, reactive oxygen species, intervertebral disc degeneration, Flexercell Tension system the level of reactive oxygen species (ROS) in NP cells induced by CMT was significantly upregulated by $3-\mathrm{MA}$. These results suggested that abnormal mechanical stress enhanced disc cell apoptosis and consequently accelerated the process of IDD. Autophagy helps to protect against CMT-induced apoptosis in disc cells and ROS may be important in this process. These findings are beneficial for further understanding the mechanism of disc cell apoptosis and autophagy.

\section{Introduction}

Lower back pain (LBP) is a common orthopedic problem in aged populations, which has a significant socio-economic effect on the lives of patients $(1,2)$. Intervertebral disc (IVD) degeneration (IDD) is considered to be the primary cause of LBP (3). IDD is characterized by histomorphological changes, including nucleus pulposus (NP) fibrosis, annulus fibrosus (AF) disorganization and cartilage endplate calcification (4-6). It has been found to be associated with several factors, including mechanical loading, aging, infection, nutrition and genetics (4). Mechanical stress is one of the important factors affecting IDD $(7,8)$.

One of the major events in IDD is the decrease of functional IVD cells (9-11). Programmed cell death (PCD) has an important role in the decrease of IVD cells. Apoptosis, also known as type I PCD, relies on the activation of caspase and is featured by DNA damage, karyopyknosis, cell shrinkage and the formation of apoptotic bodies. It has been demonstrated that apoptosis occurs in NP cells and that mechanical stress is an important factor affecting disc cell apoptosis and IDD $(12,13)$.

At present, autophagy has been widely acknowledged to be involved in several physiological and pathological processes, including maintaining cell internal homeostasis, survival, proliferation and differentiation (14). The major role of autophagy is considered to be an adaptive response of the cells to survival when faced with different stimuli, including aging, and oxidative or mechanical stress (15-17). Autophagy maintains cellular homeostasis by degrading and recycling damaged and dysfunctional organelles, proteins and other macromolecules. Various studies have demonstrated that both apoptosis and autophagy exist in IDD $(15,18-20)$. The conversion and coordination between apoptosis and autophagy remains to be fully elucidated and warrants further investigation. 
Cyclic mechanical tension (CMT), a type of mechanical loading, has been shown to influence IVD cell apoptosis and autophagy $(15,19)$. These two events share the same stimuli and regulatory proteins, but have different threshold responses to the same stimulus. The association between apoptosis and autophagy in the response of NP cells under cyclic tension has not been clarified.

In the present study, the Flexercell Tension system (Flexcell International Corporation, Hillsborough, NC, USA), a widely used system that exerts cyclic tension on cultured cells in vitro, was used for cyclic tension loading. The study illustrates how apoptosis interacts with autophagy in NP cells under cyclic tension, providing novel insight into the functions of mechanical loading in the pathophysiological significance of IDD.

\section{Materials and methods}

Ethics. The present study was approved by the Ethics Committee of Xinqiao Hospital (Chongqing, China). All protocols were subject to the standards set forth in the 8th edition of the Guide for the Care and Use of Laboratory Animals published by the National Academy of Sciences (Washington, DC, USA).

Antibodies. Rabbit monoclonal anti-rat microtubule-associated protein light chain 3 (LC3)B (cat. no. 3868, 1:1,000 dilution), Beclin-1 (cat. no. 3495, 1:1,000 dilution) and cleaved caspase-3 (cat. no. 9661, 1:1,000 dilution) were purchased from Cell Signaling Technology, Inc. (Danvers, MA, USA). Mouse monoclonal anti-rat glyceraldehyde3-phosphate dehydrogenase (GAPDH; cat. no. sc-47724, 1:1,000 dilution) was purchased from Santa Cruz Biotechnology, Inc. (Santa Cruz, CA, USA). Goat anti-rabbit horseradish peroxidase (HRP)-conjugated secondary antibody (cat. no.. ZB2301, 1:400 dilution) were purchased from ZSGB-BIO (Beijing, China).

Isolation and culture of rat NP cells. A total of 60 male Sprague-Dawley rats (age, 10-12 weeks; weight, 200-240 g; specific pathogen-free grade) were supplied by the Laboratory Animal Research Center of Daping Hospital (Chongqing, China). Animals were all housed at $20-24^{\circ} \mathrm{C}$ under a $12-\mathrm{h}$ light/dark cycle, and provided with food and water ad libitum. The caudal IVDs of 10-12-week-old male Sprague-Dawley rats were aseptically excised immediately following sacrifice. The NP tissues were carefully separated from the discs under aseptic conditions and digested in $0.2 \%$ type II collagenase (Merck KGaA, Darmstadt, Germany) at $37^{\circ} \mathrm{C}$ for $2 \mathrm{~h}$ to release the NP cells. The cell suspension was passed through a $70-\mu \mathrm{m}$ cell strainer and centrifuged at $200 \mathrm{x} \mathrm{g}$ for $5 \mathrm{~min}$ at room temperature. The supernatant was removed and the cells were suspended in DMEM/F12 medium (Thermo Fisher Scientific, Inc., Waltham, MA, USA) with $10 \%$ fetal bovine serum (Thermo Fisher Scientific, Inc.) and $1 \%$ penicillin-streptomycin (Thermo Fisher Scientific, Inc.). The NP cells were cultured in $5 \% \mathrm{CO}_{2}$ at $37^{\circ} \mathrm{C}$. The medium was replaced every 3 days. When the cells grew to $90 \%$ confluence, they were digested using $0.25 \%$ trypsin and subcultured in culture flasks. The cells of passages 2-5 were used in the subsequent experiments.

Application of CMT on NP cells. The NP cells were seeded on a 6-well BioFlex ${ }^{\mathrm{TM}}$ plate (Flexcell International Corporation,
McKeesport, PA, USA) in DMEM/F12 medium containing $10 \%$ fetal bovine serum. On reaching $80 \%$ confluence, the NP cells were stretched with CMT (20\% elongation) at a frequency of $1 \mathrm{~Hz}$ for 2, 4, 6, 12, 24 or $48 \mathrm{~h}$ using a FX-5000T Flexercell Tension Plus system (Flexcell International Corporation) at $37^{\circ} \mathrm{C}$ and $5 \% \mathrm{CO}_{2}$. Cells cultured in the same plates under the same conditions were used as the control. Following cyclic tension loading, the morphology of NP cells was observed using a phase contrast microscope (Olympus Corporation, Tokyo, Japan).

Reverse transcription-quantitative polymerase chain reaction (RT-qPCR) analysis. Total RNA was isolated from the NP cell using TRIzol reagent (Takara Bio, Inc., Otsu, Japan). The RNA quality and quantity were determined using a NanoDrop ND-1000 spectrophotometer (Thermo Fisher Scientific, Inc). RNA $(1 \mu \mathrm{g})$ was reverse transcribed using a PrimeScript RT Reagent kit (Takara Bio). The PrimeScript RT Reagent kit (Takara Bio, Inc.) was used for RT-qPCR according to the manufacturer's instructions. RT-qPCR was performed using a ViiA ${ }^{\mathrm{TM}} 7$ Real-Time PCR system using SYBR ${ }^{\circledR}$ Premix Ex $\mathrm{Taq}^{\mathrm{TM}}$ II (Takara Bio, Inc.), according to the manufacturer's instructions. The 20- $\mu$ l reaction volume was amplified under the following conditions: Initial heat activation for $30 \mathrm{sec}$ at $95^{\circ} \mathrm{C}$, followed by 40 cycles of $5 \mathrm{sec}$ at $95^{\circ} \mathrm{C}$ for template denaturation and $30 \mathrm{sec}$ at $60^{\circ} \mathrm{C}$ for annealing and extension. GAPDH was used as an internal reference gene. Data were analyzed using the $2^{-\Delta \Delta C q}$ method (21). The primers of the genes investigated in the present study are listed in Table I.

Western blot analysis. Total proteins were extracted from NP cells using radioimmunoprecipitation assay (RIPA) lysis buffer (Thermo Fisher Scientific, Inc.). The concentration of protein was quantified using the bicinchoninic acid method (Beyotime Institute of Biotechnology). Protein samples $(50 \mu \mathrm{g})$ were separated using 10\% SDS-PAGE and transferred onto polyvinylidene difluoride membranes (EMD Millipore, Billerica, MA, USA). The membranes were blocked with 5\% milk in TBST for $1 \mathrm{~h}$ at $37^{\circ} \mathrm{C}$ to block nonspecific protein binding. The membranes were then incubated at $4^{\circ} \mathrm{C}$ overnight with primary antibodies, following which the membranes were washed with TBST and incubated at $37^{\circ} \mathrm{C}$ for $1 \mathrm{~h}$ with HRP-conjugated secondary antibodies. Immunolabeling was detected using ECL reagents (Thermo Fisher Scientific, Inc.). The optical density of the bands was measured using Image $\mathbf{J}$ software (National Institutes of Health).

Transmission electron microscopy (TEM). Following stretching, the NP cells were isolated with trypsin and fixed in $2 \%$ glutaraldehyde at $4^{\circ} \mathrm{C}$ for 2 days. The cells were then treated with $1 \%$ osmium tetroxide (Sigma-Aldrich; Merck KGaA) for $30 \mathrm{~min}$ at room temperature, dehydrated in an ethanol gradient and embedded with Epon 812 (Shell Chemical Co., Houston, TX, USA). The fixed cells were then sliced into ultrathin sections $(60 \mathrm{~nm})$ and observed under a Tecnai-10 TEM (Philips Healthcare, Amsterdam, The Netherlands).

Apoptotic incidence measurement. The apoptotic incidence was detected using the Annexin V-FITC apoptosis detection kit I (BD Biosciences, San Jose, CA, USA) according to the 
Table I. Primer sequences used in reverse transcription-quantitative polymerase chain reaction analysis.

\begin{tabular}{lll}
\hline Target gene & \multicolumn{1}{c}{ Forward primer (5'-3') } & \multicolumn{1}{c}{ Reverse primer (5'-3') } \\
\hline LC3 & TCACGGACGGAAGCCAACACA & AATCCTTCCCGACCGCACCAT \\
Beclin-1 & ACAGCGGACAATTTGGCACGAT & TGGAGCAACAACACTGTCTGGC \\
Caspase-3 & GCACACGGGACTTGGAAAGCAT & AGCGATGACTCAGCACCTCCAT \\
GAPDH & CCAGCAAGAGCACAAGAGGAAGAG & GGTCTACATGGCAACTGTGAGGAG \\
\hline
\end{tabular}

LC3, microtubule-associated protein light chain 3; GAPDH, glyceraldehyde3-phosphate dehydrogenase.

manufacturer's instructions. Following stretching, the NP cells were isolated with trypsin and washed twice with PBS. The NP cells were then resuspended in $500 \mu 1$ binding buffer at a concentration of $1 \times 10^{6}$ cells $/ \mathrm{ml}$. PE Annexin V solution $(5 \mu \mathrm{l})$ and 7-amino-actinomycin D (5 $\mu \mathrm{l})$ were added to the cells at room temperature for $15 \mathrm{~min}$ in the dark. Finally, the cells were analyzed on a flow cytometer (Beckman Coulter, Inc., Brea, CA, USA).

Autophagy incidence measurement. Following stretching, the NP cells were isolated with trypsin and washed twice with PBS, resuspended in PBS with $0.05 \mathrm{mM}$ monodansylcadaverine (Merck KGaA) at a concentration of $1 \times 10^{6}$ cells $/ \mathrm{ml}$, and incubated at $37^{\circ} \mathrm{C}$ and $5 \% \mathrm{CO}_{2}$ for $20 \mathrm{~min}$ in the dark. Following incubation, the cells were washed three times with PBS. The mean fluorescence intensity (MFI) was analyzed on a flow cytometer (Beckman Coulter, Inc.).

Reactive oxygen species (ROS) measurement. Following stretching, the NP cells were isolated with trypsin and washed twice with PBS. The cells were then resuspended using serum-free DMEM/F12 medium with $25 \mu \mathrm{M}$ 2',7'-dichlorodihydrofluorescein diacetate (Merck $\mathrm{KGaA}$ ) at a concentration of $1 \times 10^{6}$ cells $/ \mathrm{ml}$, and incubated at $37^{\circ} \mathrm{C}$ and $5 \% \mathrm{CO}_{2}$ for $20 \mathrm{~min}$. Following incubation, the cells were washed three times with serum-free DMEM/F12 medium. The MFI was analyzed on a flow cytometer (Beckman Coulter, Inc.).

Statistical analysis. Data are presented as the mean \pm standard deviation. All experiments were repeated at least three times. For comparisons between two independent groups, Student's t-test was used. For comparisons among three or more groups, one-way ANOVA and least significant difference multiple comparisons were used. The RT-qPCR data were analyzed using Kruskal-Wallis nonparametric analysis and Mann-Whitney U post hoc tests. Data were analyzed and displayed using SPSS version 22.0 (International Business Machines Corp., Armonk, NY, USA) and GraphPad Prism 6 software (GraphPad Software Inc., La Jolla, CA, USA). P<0.05 was considered to indicate a statistically significant difference.

\section{Results}

Morphology of NP cells following CMT application. Following cyclic tension loading, the morphology of NP cells was observed using a phase contrast microscope. The NP cells that adhered to the outer part of the membrane became clearly spindle-shaped and exhibited a circular arrangement perpendicular to the axial tension (Fig. 1). Only a few cells were detached from the membrane surface.

Effect of CMT on apoptosis of rat NP cells. Following $20 \%$ CMT at a frequency of $1 \mathrm{~Hz}$ for $6,12,24$ and $48 \mathrm{~h}$, the apoptotic rate of the NPs was detected by flow cytometry with Annexin $\mathrm{V}$ and 7AAD double-labeling. The results demonstrated a time-dependent increase in the apoptotic rate of rat NP cells subjected to CMT (Fig. 2A), and significant differences in the apoptotic rate were observed after $24 \mathrm{~h}$ (Fig. 2B). Consistent with the results of flow cytometry, the western blot results showed that the levels of caspase- 3 and cleaved caspase-3 were also increased in the NP cells subjected to CMT (Fig. 2C-E).

Effect of CMT on the autophagy of rat NP cells. Examining the ultrastructure of cells by TEM is the gold standard for the detection of autophagy (14). The ultrastructure of the NP cells was observed under TEM. The autophagosomes, manifested as double-membrane vacuolar structures containing organelles and parts of cytoplasm, were observed in NP cells. The number of autophagosomes in the CMT groups was higher than the number in the static control groups (Fig. 3). MDC staining and a flow cytometry quantitative assay were used to quantify the expression of autophagy. The increase in MFI in each CMT group was measured. There was a time-dependent decrease in the MFI of NP cells subjected to 20\% CMT for 6, 12, 24 and $48 \mathrm{~h}$ (Fig. 4A). The mRNA expression levels of LC3 and Beclin-1 showed a similar trend (Fig. 4B and C). The protein expression of LC3 II and Beclin-1 was also increased significantly in the NP cells subjected to CMT for $6 \mathrm{~h}$ (Fig. 4D-F), and exhibited a time-dependent decrease after $6 \mathrm{~h}$. These results indicated the upregulation of autophagy in NP cells subjected to CMT. Furthermore, the protein expression of LC3 II was measured at 2, 4 and $6 \mathrm{~h}$ to determine when autophagy was the most marked. The results showed that autophagy increased within $6 \mathrm{~h}$ and reached a maximum level between 4 and $6 \mathrm{~h}$ (Fig. S1).

Autophagy is modulated by rapamycin and 3-MA in NP cells subjected to CMT. The NP cells were administered with rapamycin $(1 \mu \mathrm{M})$ or 3-MA $(100 \mathrm{nM})$ and placed in an incubator for $1 \mathrm{~h}$, following which the NP cells were loaded with $20 \% \mathrm{CMT}$ for $24 \mathrm{~h}$. The results of MDC staining and quantitative fluorescence measured by flow cytometry indicated that the activities of autophagy in cells following CMT loading 


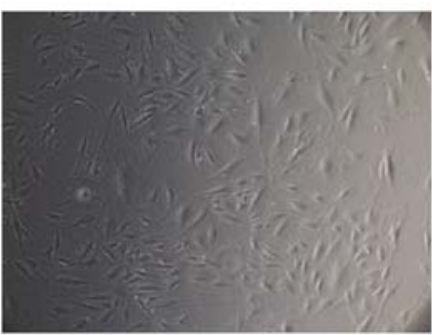

Control

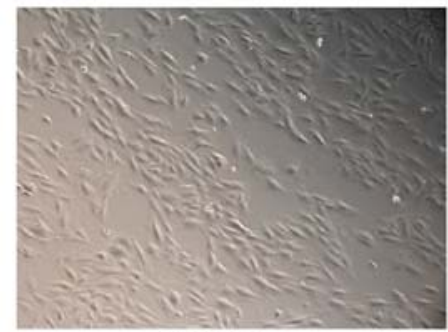

CMT-6 h

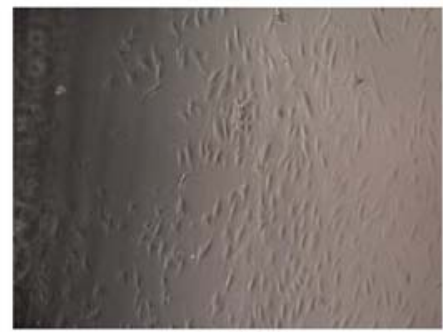

CMT-12 h

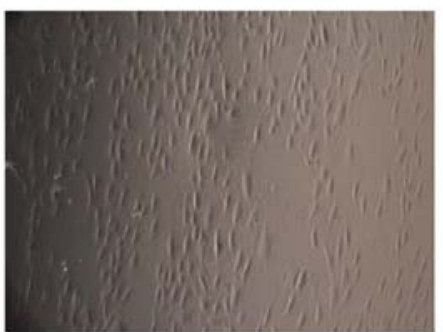

CMT-24 h

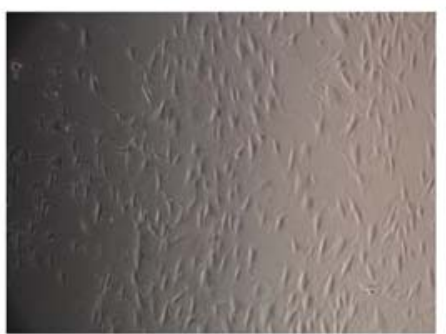

CMT-48 h

Figure 1. Morphology of NP cells subjected to CMT with 20\% elongation for 6, 12, 24 and $48 \mathrm{~h}$ (magnification, x100). The control comprised NP cells that were cultured under the same conditions and kept static. NP, nucleus pulposus; CMT, cyclic mechanical tension.
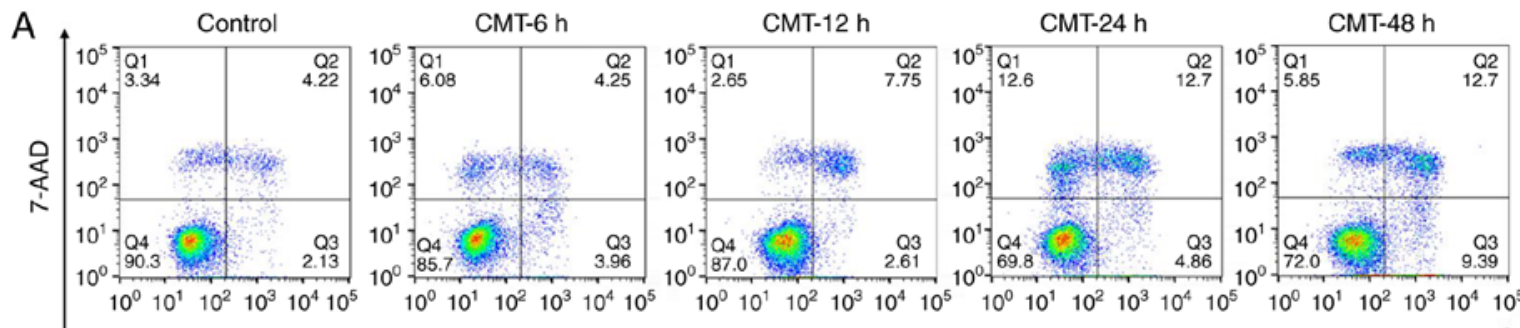

B

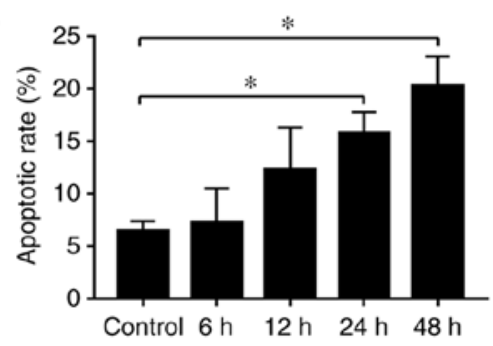

$\mathrm{D}$

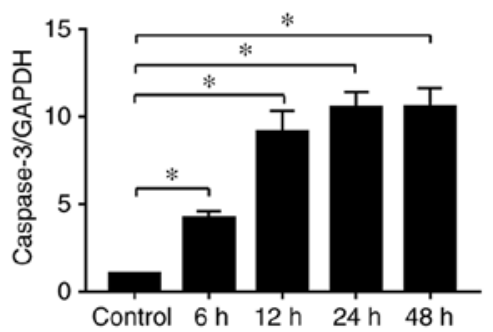

Annexin V-FITC

$\mathrm{C}$

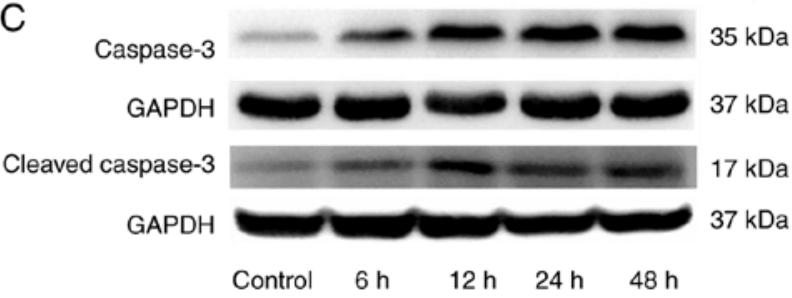

E

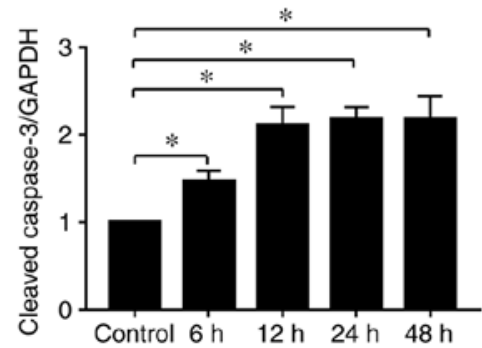

Figure 2. Effects of CMT on NP cell apoptosis. (A) Representative graph of cell apoptosis obtained by flow cytometry following double-labeling with Annexin V/7-AAD. (B) Statistical analysis of the apoptotic rate. (C) Representative western blot images and quantification of (D) caspase-3 and (E) cleaved caspase-3 protein expression in NP cells subjected to CMT. The control comprised NP cells kept static. ${ }^{\mathrm{P}}<0.05$. Error bars represent the standard error. NP, nucleus pulposus; FITC, fluorescein isothiocyanate; CMT, cyclic mechanical tension; 7-AAD, 7-amino-actinomycin D; GAPDH, glyceraldehyde3-phosphate dehydrogenase.

were significantly reduced by 3 -MA $(\mathrm{P}<0.05$; Fig. 5A). The difference in autophagic activities between the CMT and the combined CMT and rapamycin was not significant $(\mathrm{P}=0.39)$. The mRNA expression levels of LC3 and Beclin-1 were decreased by 3 -MA in NP cells subjected to $20 \%$ CMT for
$24 \mathrm{~h}$, but did not differ significantly following combined CMT and rapamycin treatment (Fig. 5B). Consistent with the results of the RT-qPCR analysis, the western blot analysis results confirmed the downregulation of LC3 and Beclin-1 by 3-MA at the protein level (Fig. 5C and D). 


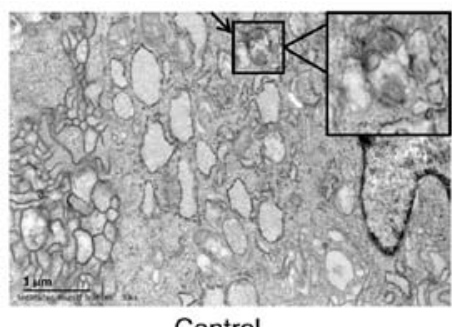

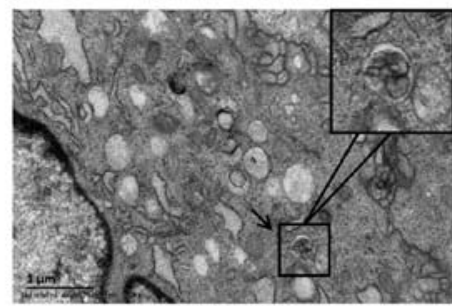

CMT-6 h

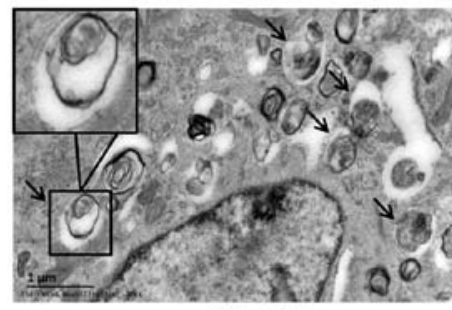

CMT-12 h

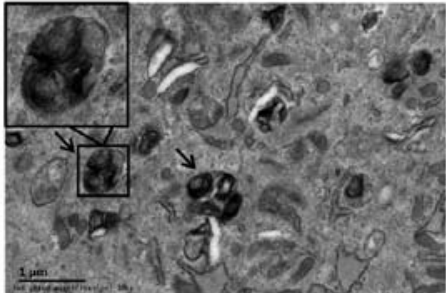

CMT-24 h

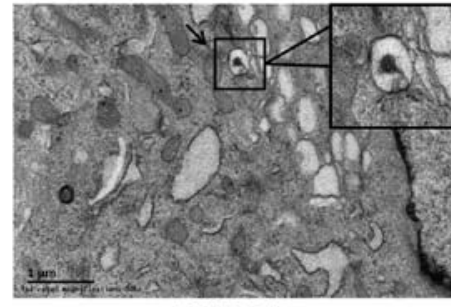

CMT-48 h

Figure 3. Ultrastructure of NP cells subjected to CMT and observed by transmission electron microscopy. Autophagosomes are marked by arrowheads. Typical autophagosomes are shown at a magnification, x60,000. NP cells kept static were used as the control. NP, nucleus pulposus; CMT, cyclic mechanical tension.

A

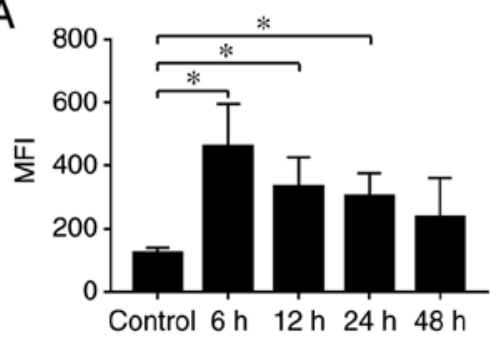

D

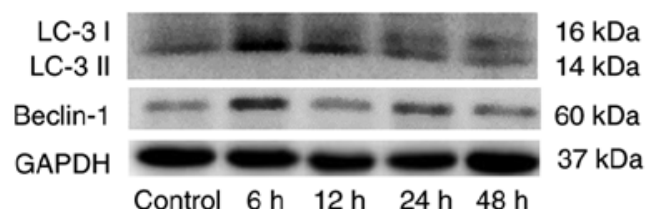

B
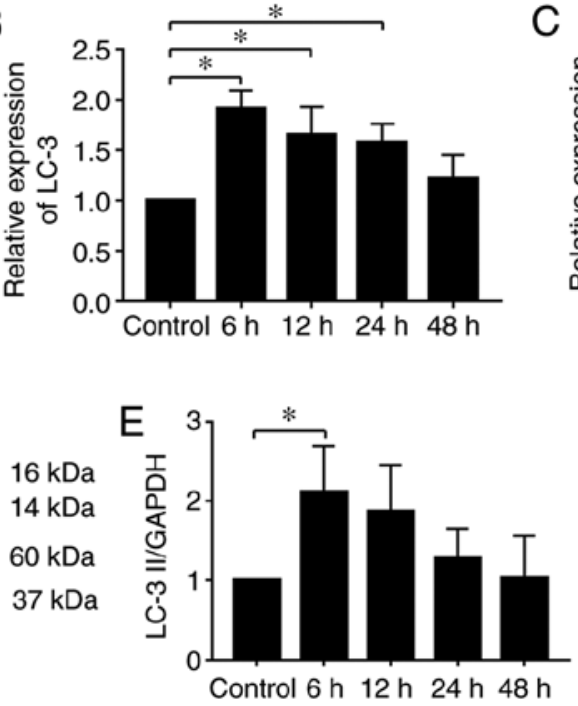

C
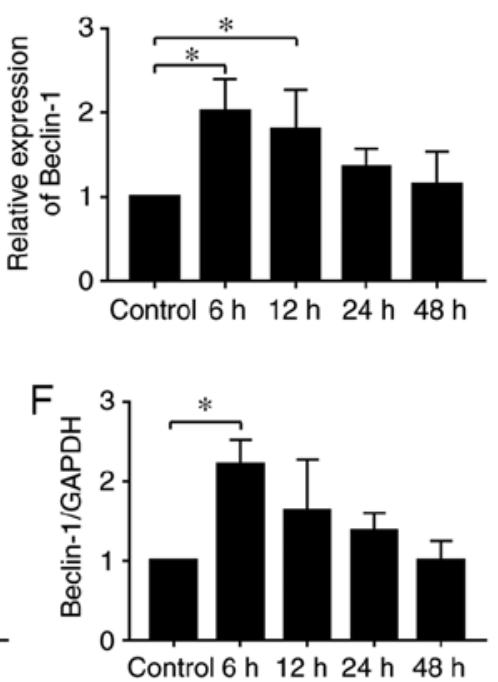

Figure 4. Effects of CMT on autophagy in NP cells. (A) Levels of autophagy in NP cells were measured by flow cytometry with monodansylcadaverine staining. Expression levels of (B) LC3 and (C) Beclin-1 were detected by reverse transcription quantitative polymerase chain reaction and (D) western blotting. Protein levels of (E) LC3-II and (F) Beclin-1. The control comprised NP cells that were kept static. "P<0.05. Error bars represent the standard error. NP, nucleus pulposus; CMT, cyclic mechanical tension; MFI, mean fluorescence intensity; LC3, microtubule-associated protein light chain 3; GAPDH, glyceraldehyde3-phosphate dehydrogenase.

ROS levels in NP cells subjected to $20 \%$ CMT are increased by 3-MA. Our previous study indicated that CMT had minimal effect on the production of ROS in NP cells (22). In the present study, the ROS levels of NP cells pretreated with 3-MA or rapamycin following CMT were measured at $24 \mathrm{~h}$. The results showed that the level of ROS in NP cells was increased significantly in the combined CMT and 3-MA group $(\mathrm{P}<0.05)$, with no significant differences observed among other groups (Fig. 6). In order to further illustrate the effect of autophagy on ROS production, ROS levels were measured at $6 \mathrm{~h}$ when autophagy was the highest (Fig. S2). The results showed that the level of ROS in NP cells increased significantly with $3-\mathrm{MA}$ treatment at $6 \mathrm{~h}(\mathrm{P}<0.05)$.

Apoptosis of NP cellsloaded with 20\% CMTisinduced by 3-MA. The NP cells were loaded with $20 \%$ CMT for $24 \mathrm{~h}$ following incubation with $1 \mu \mathrm{M}$ rapamycin or $100 \mathrm{nM} 3-\mathrm{MA}$ for $1 \mathrm{~h}$. The apoptotic rates of NP cells were then measured (Fig. 7A). Compared with the CMT group, the apoptotic rate of NP cells was significantly increased in the combined CMT and 3-MA group $(\mathrm{P}<0.05)$, with no clear differences observed in the combined CMT and rapamycin group (Fig. 7B). Changes in the protein expression of caspase- 3 and cleaved caspase- 3 were similar (Fig. 7C-E). According to the results, autophagy was highest following application of CMT for $6 \mathrm{~h}$. To verify the inhibitory effect of autophagy on apoptosis, the apoptotic rates and expression of cleaved caspase- 3 were measured in NP cells treated with 3-MA and CMT at $6 \mathrm{~h}$ (Fig. S3). The apoptotic rate of NP cells and the protein expression of cleaved caspase- 3 were significantly increased in the combined CMT and 3-MA group at $6 \mathrm{~h}(\mathrm{P}<0.05)$. 
A

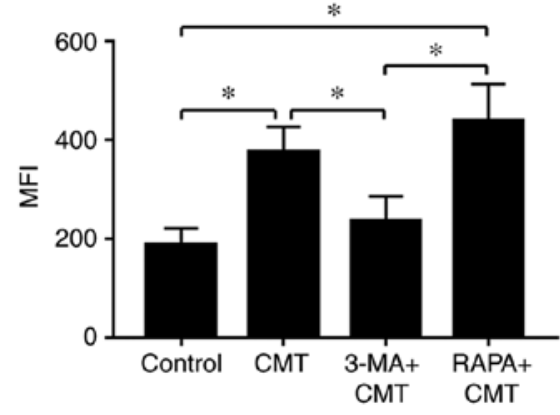

C

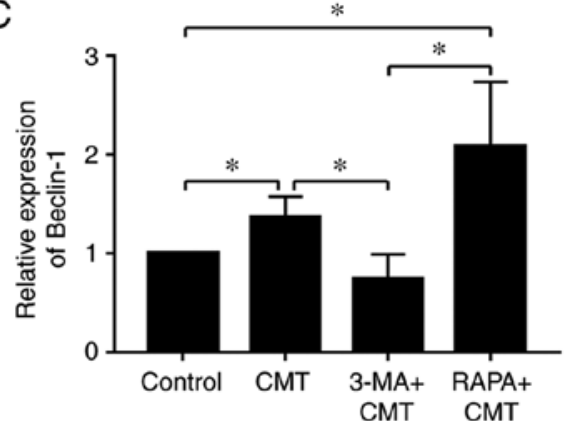

B

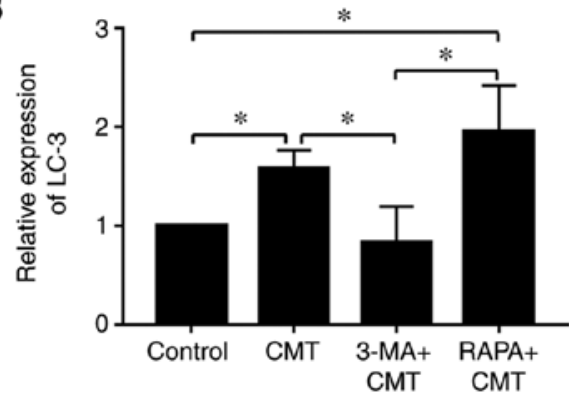

D

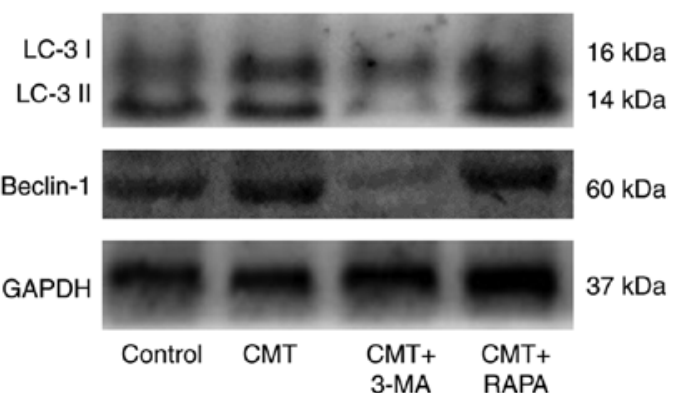

Figure 5. Effects of autophagy inhibitor 3-MA and inducer rapamycin on autophagy in NP cells subjected to CMT for 24 h. (A) Autophagy in NP cells was measured by flow cytometry with monodansylcadaverine staining. Expression levels of (B) LC3 and (C) Beclin-1 were detected by reverse-transcription quantitative polymerase chain reaction and (D) western blotting. The control comprised NP cells that were kept static. ${ }^{*} \mathrm{P}<0.05$. Error bars represent the standard error. NP, nucleus pulposus; MFI, mean fluorescence intensity; RAPA, rapamycin; CMT, cyclic mechanical tension; LC3, microtubule-associated protein light chain 3; GAPDH, glyceraldehyde3-phosphate dehydrogenase.

\section{Discussion}

IVDs are basic structures in the human body that are important in spinal movement. Studies have demonstrated that the initiation of IDD is associated with the degradation of extracellular matrix and changes in the behavior of IVD cells, including senescence, necrosis and apoptosis $(11,23)$. NP cells, a major group of IVD cells, are exposed to various mechanical stresses, including tension (8). The loss of NP cells has been closely correlated with the onset of IDD (9). Apoptosis, also known as type I PCD, has been shown to be important in the decrease of NP cells (24). Previous studies have indicated that excessive stretching induced the apoptosis of rat AF cells $(19,25)$ and cartilage endplate-derived stem cells (18). The present study showed that $20 \%$ CMT, which is considered excessive stretch towards NP cells, induced apoptosis in rat NP cells, with the incidence of apoptosis increasing with the increasing duration of CMT. The results indicated that apoptosis induced by prolonged excessive mechanical stress in NP cells may be one of the factors causing a decrease in the number of functional disc cells, consequently accelerating the initiation and progression of IDD.

Autophagy is an adaptive response of cells to survival when faced with different stimuli, including aging and oxidative or mechanical stress. Autophagy is a conserved and ubiquitous physiological mechanism that can degrade damaged macromolecules and organelles to maintain cellular homeostasis (26). The role of autophagy in IDD has been widely investigated. Ye et al demonstrated that autophagy existed in rat NP cells (27). Furthermore, it has been demonstrated that autophagy is a protective mechanism against apoptosis in podocytes and AF cells $(28,29)$. However, the association

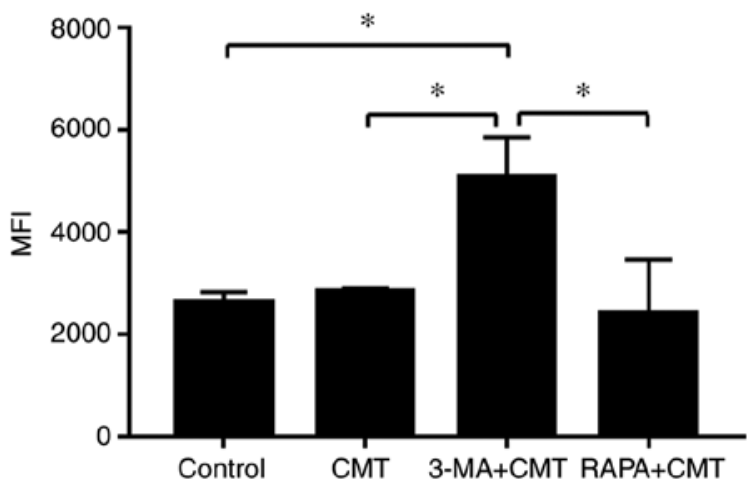

Figure 6. Effects of autophagy inhibitor 3-MA and inducer rapamycin on intracellular ROS of NP cells subjected to CMT for $24 \mathrm{~h}$. The level of intracellular ROS was detected by flow cytometry using $2^{\prime}, 7^{\prime}$-dichlorodihydrofluorescein diacetate. ${ }^{*} \mathrm{P}<0.05$. Error bars represent the standard error. The control comprised NP cells that were kept static. ROS, reactive oxygen species; NP, nucleus pulposus; MFI, mean fluorescence intensity; CMT, cyclic mechanical tension.

between autophagy and CMT-induced apoptosis in NP cells has remained unclear.

In the present study, autophagy was observed in NP cells and was upregulated by $20 \%$ CMT. The increasing level of autophagy decreased in a time-dependent manner following the application of CMT to NP cells for $6 \mathrm{~h}$, and the incidence of apoptosis in NP cells increased as the duration of CMT increased. The results suggested that autophagy may be a protective mechanism against apoptosis induced by CMT in NP cells. Therefore, the association between autophagy and stretch-induced apoptosis in NP cells was investigated. Rapamycin and 3-MA were used to regulate autophagy in the 
A

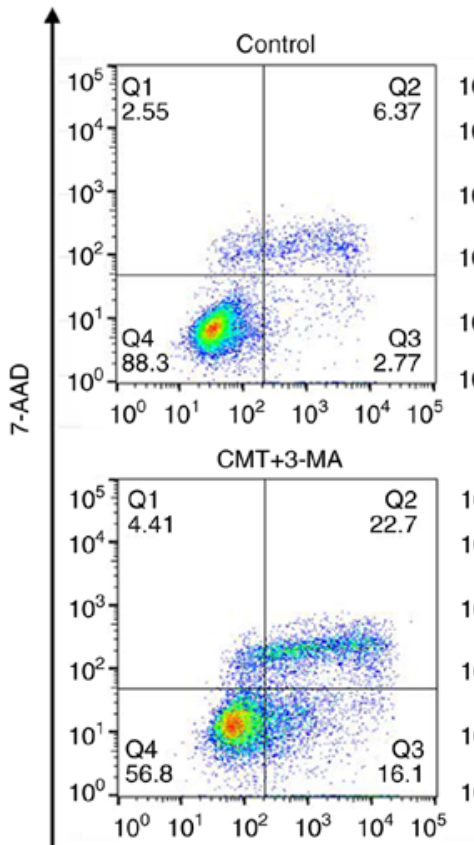

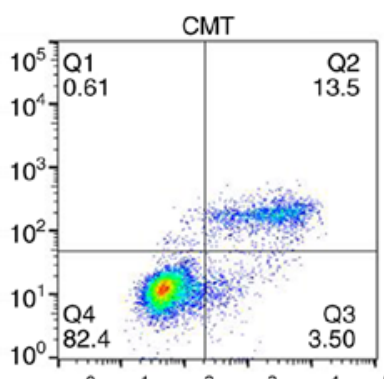
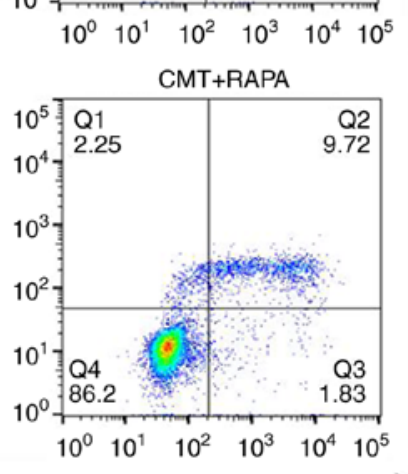

B

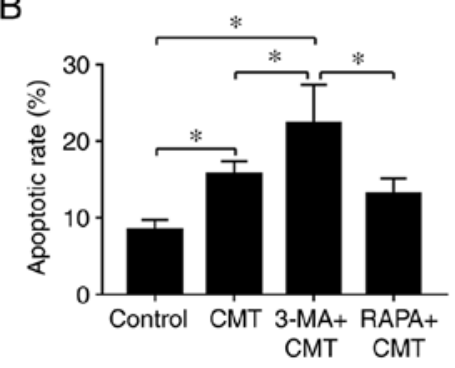

Annexin V-FITC

C

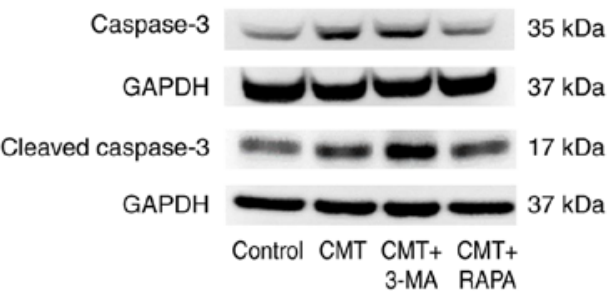

D

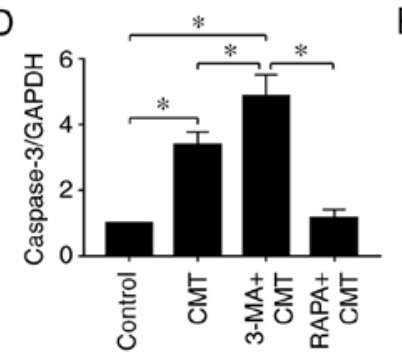

E

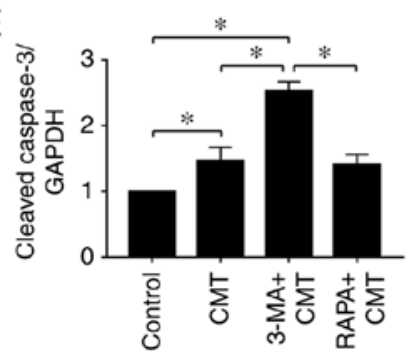

Figure 7. Effects of autophagy inhibitor 3-MA and inducer rapamycin on cell apoptosis of NP cells subjected to CMT for 24 h. (A) Representative graph of cell apoptosis obtained by flow cytometry following double-labeling with Annexin V/7-AAD. (B) Statistical analysis of the apoptotic rate. (C) Representative western blot images and quantification of protein expression levels of (D) caspase-3 and (E) cleaved caspase-3 in NP cells subjected to CMT. The control comprised NP cells that were kept static. ${ }^{*} \mathrm{P}<0.05$. Error bars represent the standard error. NP, nucleus pulposus; CMT, cyclic mechanical tension; 7-AAD, 7-amino-actinomycin D; FITC, fluorescein isothiocyanate; RAPA, rapamycin.

NP cells. The results showed that autophagy was significantly inhibited by 3-MA in NP cells subjected to CMT, whereas rapamycin had minimal effect. The group treated with 3-MA, an autophagy inhibitor, exhibited a significant decrease in the incidence of autophagy following $24 \mathrm{~h}$ of CMT treatment, whereas an increase in the incidence of apoptosis was noted. Rapamycin, an autophagy promoter, had no significant effect on autophagy. In conclusion, the inhibition of autophagy causes an increase in the incidence of apoptosis in NP cells subjected to CMT. The results suggested that autophagy protects against CMT-induced apoptosis in NP cells. Furthermore, the underlying mechanism of autophagy in the protection against CMT-induced apoptosis of NP cells warrants further investigation.

Oxidative stress caused by excessive ROS generation is also an essential trigger of NP cell apoptosis (30). It has been reported that ROS can trigger autophagy in cancer cells as signaling molecules (16). In turn, autophagy may contribute to reducing the generation of intracellular ROS and oxidative damage by clearing oxidized macromolecules and organelles (20). Our previous study suggested that CMT had little effect on the production of ROS in NP cells. However,
NP cells grow in a low oxygen environment in normal IVD, and cultured NP cells in vitro grow in a high oxygen environment $(31,32)$. ROS was overgenerated by high oxygen stimuli following isolation of the NP cells from the tissue and culture in vitro (33). This may be the reason for CMT having little effect on the production of ROS in NP cells in our previous study. In contrast with our previous study, the present study found that the ROS level was significantly increased in the group in which autophagy was inhibited by 3-MA; apoptosis in NP cells was also enhanced. In combination, the results of the present study suggested that ROS serve an important role in CMT-induced apoptosis, and that autophagy protects against apoptosis by reducing the generation of intracellular ROS and oxidative damage in NP cells. Details of the functions and mechanisms underlying the associations among ROS, autophagy and CMT-induced apoptosis require further investigation.

In conclusion, the present study suggested that the effect of CMT on NP cell apoptosis is duration-dependent. In addition, autophagy protected against CMT-induced apoptosis in NP cells in vitro, with ROS potentially having an important role in this process. These results suggest that maintaining the 
autophagy level of disc cells is conducive to alleviating apoptosis caused by mechanical stress and may assist in delaying the process of IDD. However, the cellular biological response to mechanical stress depends on different factors, including the duration, frequency and magnitude of mechanical stress, in addition to the disc cell type $(22,34,35)$, and further experiments are warranted to clarify these issues.

\section{Acknowledgements}

Not applicable.

\section{Funding}

The present study was funded by the National Natural Science Foundation of China (grant nos. 81672215, 81572186, 81702182 and 81874028).

\section{Availability of data and material}

All data generated or analyzed during this study are included in this published article.

\section{Authors' contributions}

MY contributed to the conception and design of the study, collection, analysis and interpretation of data and writing of the manuscript. CF contributed to the conception and design of the study, and writing and revision of the manuscript. YZ contributed to the collection of data and provision of study material. CL contributed to the collection of data and provision of study material; BL contributed to the collection, analysis and interpretation of data; QZ contributed to the collection, analysis and interpretation of data; $\mathrm{BH}$ contributed to the conception and design and final approval of the manuscript; YZ contributed to the conception and design of the study, final approval of the version to be published and financial and administrative support. All authors read and approved the final manuscript.

\section{Ethics approval and consent to participate}

This study was approved by the Ethics Committee of Xinqiao Hospital. All protocols described in current study were in accordance with the standards set forth in the eighth edition of Guide for the Care and Use of Laboratory Animals published by the National Academy of Sciences.

\section{Patient consent for publication}

Not applicable.

\section{Competing interests}

The authors declare that they have no competing interests.

\section{References}

1. Haldeman S, Kopansky-Giles D, Hurwitz EL, Hoy D, Mark Erwin W, Dagenais S, Kawchuk G, Strömqvist B and Walsh N: Advancements in the management of spine disorders. Best Pract Res Clin Rheumatol 26: 263-280, 2012.
2. Meucci RD, Fassa AG and Faria NM: Prevalence of chronic low back pain: Systematic review. Rev Saude Publica 49, 2015.

3. Cheung KM: The relationship between disc degeneration, low back pain, and human pain genetics. Spine J 10: 958-960, 2010.

4. Roberts S, Evans H, Trivedi J and Menage J: Histology and pathology of the human intervertebral disc. J Bone Joint Surg Am 88 (Suppl 2): S10-S14, 2006.

5. Kadow T, Sowa G, Vo N and Kang JD: Molecular basis of intervertebral disc degeneration and herniations: What are the important translational questions? Clin Orthop Relat Res 473: 1903-1912, 2015.

6. Kepler CK, Ponnappan RK, Tannoury CA, Risbud MV and Anderson DG: The molecular basis of intervertebral disc degeneration. Spine J 13: 318-330, 2013.

7. Setton LA and Chen J: Mechanobiology of the intervertebral disc and relevance to disc degeneration. J Bone Joint Surg Am 88 (Suppl 2): S52-S57, 2006.

8. Vergroesen PP, Kingma I, Emanuel KS, Hoogendoorn RJ, Welting TJ, van Royen BJ, van Dieën JH and Smit TH: Mechanics and biology in intervertebral disc degeneration: A vicious circle. Osteoarthritis Cartilage 23: 1057-1070, 2015.

9. Ding F, Shao ZW and Xiong LM: Cell death in intervertebral disc degeneration. Apoptosis 18: 777-785, 2013.

10. Zhang F, Zhao X, Shen H and Zhang C: Molecular mechanisms of cell death in intervertebral disc degeneration (Review). Int J Mol Med 37: 1439-1448, 2016.

11. Zhao CQ, Jiang LS and Dai LY: Programmed cell death in intervertebral disc degeneration. Apoptosis 11: 2079-2088, 2006.

12. Ariga K, Yonenobu K, Nakase T, Hosono N, Okuda S, Meng W, Tamura Y and Yoshikawa H: Mechanical stress-induced apoptosis of endplate chondrocytes in organ-cultured mouse intervertebral discs: An ex vivo study. Spine (Phila Pa 1976) 28: 1528-1533, 2003.

13. Ma JF, Zang LN, Xi YM, Yang WJ and Zou D: MiR-125a Rs12976445 polymorphism is associated with the apoptosis status of nucleus pulposus cells and the risk of intervertebral disc degeneration. Cell Physiol Biochem 38: 295-305, 2016.

14. Hönscheid P, Datta K and Muders MH: Autophagy: Detection, regulation and its role in cancer and therapy response. Int $\mathrm{J}$ Radiat Biol 90: 628-635, 2014.

15. Xu HG, Yu YF, Zheng Q, Zhang W, Wang CD, Zhao XY, Tong WX, Wang H, Liu P and Zhang XL: Autophagy protects end plate chondrocytes from intermittent cyclic mechanical tension induced calcification. Bone 66: 232-239, 2014.

16. Li L, Tan J, Miao Y, Lei P and Zhang Q: ROS and autophagy: Interactions and molecular regulatory mechanisms. Cell Mol Neurobiol 35: 615-621, 2015.

17. Kang C, Xu Q, Martin TD, Li MZ, Demaria M, Aron L, Lu T, Yankner BA, Campisi J and Elledge SJ: The DNA damage response induces inflammation and senescence by inhibiting autophagy of GATA4. Science 349: aaa5612, 2015.

18. Yuan C, Pu L, He Z and Wang J: BNIP3/Bcl-2-mediated apoptosis induced by cyclic tensile stretch in human cartilage endplate-derived stem cells. Exp Ther Med 15: 235-241, 2018.

19. Zhang YH,Zhao CQ, Jiang LS and Dai LY: Cyclic stretch-induced apoptosis in rat annulus fibrosus cells is mediated in part by endoplasmic reticulum stress through nitric oxide production. Eur Spine J 20: 1233-1243, 2011.

20. Ma KG, Shao ZW, Yang SH, Wang J, Wang BC, Xiong LM, Wu Q and Chen SF: Autophagy is activated in compression-induced cell degeneration and is mediated by reactive oxygen species in nucleus pulposus cells exposed to compression. Osteoarthritis Cartilage 21: 2030-2038, 2013.

21. Livak KJ and Schmittgen TD: Analysis of relative gene expression data using real-time quantitative PCR and the 2(-Delta Delta C(T)) method. Methods 25: 402-408, 2001.

22. Feng C, Yang M, Zhang Y, Lan M, Huang B, Liu H and Zhou Y: Cyclic mechanical tension reinforces DNA damage and activates the p53-p21-Rb pathway to induce premature senescence of nucleus pulposus cells. Int J Mol Med 41: 3316-3326, 2018.

23. Vo NV, Hartman RA, Patil PR, Risbud MV, Kletsas D, Iatridis JC, Hoyland JA, Le Maitre CL, Sowa GA and Kang JD: Molecular mechanisms of biological aging in intervertebral discs. J Orthop Res 34: 1289-1306, 2016.

24. Gruber HE and Hanley EN Jr: Analysis of aging and degeneration of the human intervertebral disc. Comparison of surgical specimens with normal controls. Spine (Phila Pa 1976) 23: 751-757, 1998.

25. Rannou F, Lee TS, Zhou RH, Chin J, Lotz JC, MayouxBenhamou MA, Barbet JP, Chevrot A and Shyy JY: Intervertebral disc degeneration: The role of the mitochondrial pathway in annulus fibrosus cell apoptosis induced by overload. Am J Pathol 164: 915-924, 2004. 
26. Levine B and Kroemer G: Autophagy in the pathogenesis of disease. Cell 132: 27-42, 2008.

27. Ye W, Xu K, Huang D, Liang A, Peng Y, Zhu W and Li C: Age-related increases of macroautophagy and chaperone-mediated autophagy in rat nucleus pulposus. Connect Tissue Res 52: 472-478, 2011

28. Shen C, Yan J, Jiang LS and Dai LY: Autophagy in rat annulus fibrosus cells: Evidence and possible implications. Arthritis Res Ther 13: R132, 2011.

29. Dong C, Zheng H, Huang S, You N, Xu J, Ye X, Zhu Q, Feng Y, You Q, Miao H, et al: Heme oxygenase-1 enhances autophagy in podocytes as a protective mechanism against high glucose-induced apoptosis. Exp Cell Res 337: 146-159, 2015.

30. Sinha K, Das J, Pal PB and Sil PC: Oxidative stress: The mitochondria-dependent and mitochondria-independent pathways of apoptosis. Arch Toxicol 87: 1157-1180, 2013.

31. Chen JW, Ni BB, Zheng XF, Li B, Jiang SD and Jiang LS: Hypoxia facilitates the survival of nucleus pulposus cells in serum deprivation by down-regulating excessive autophagy through restricting ROS generation. Int J Biochem Cell Biol 59: 1-10, 2015.
32. Wang F, Shi R, Cai F, Wang YT and Wu XT: Stem cell approaches to intervertebral disc regeneration: Obstacles from the disc microenvironment. Stem Cells Dev 24: 2479-2495, 2015.

33. Feng C, Zhang Y, Yang M, Lan M, Liu H, Huang B and Zhou Y: Oxygen-sensing Nox4 generates genotoxic ROS to induce premature senescence of nucleus pulposus cells through MAPK and NF-кB pathways. Oxid Med Cell Longev 2017: 7426458, 2017.

34. Neidlinger-Wilke C, Galbusera F, Pratsinis H, Mavrogonatou E, Mietsch A, Kletsas D and Wilke HJ: Mechanical loading of the intervertebral disc: From the macroscopic to the cellular level. Eur Spine J 23 (Suppl 3): S333-S343, 2014.

35. Daly C, Ghosh P, Jenkin G, Oehme D and Goldschlager T: A review of animal models of intervertebral disc degeneration: Pathophysiology, regeneration, and translation to the clinic. Biomed Res Int 2016: 5952165, 2016. 